\title{
More advanced disease and worse survival in cryptogenic compared to viral hepatocellular carcinoma
}

\author{
Tomi W. Jun ${ }^{1}$ | Ming-Lun Yeh ${ }^{2}$ | Ju Dong Yang ${ }^{3}$ | Vincent L. Chen ${ }^{1,4}$ | Pauline Nguyen ${ }^{1}$ | \\ Nasra H. Giama ${ }^{3}$ | Chung-Feng Huang ${ }^{2}$ | Ann W. Hsing ${ }^{5}$ | Chia-Yen Dai ${ }^{2}$ | \\ Jee-Fu Huang ${ }^{2}$ | Wan-Long Chuang ${ }^{2}$ | Lewis R. Roberts ${ }^{3}$ | Ming-Lung $\mathrm{Yu}^{2}$ | \\ Mindie H. Nguyen ${ }^{1}$
}

${ }^{1}$ Division of Gastroenterology and Hepatology, Stanford University Medical Center, Stanford, CA, USA

${ }^{2}$ Hepatobiliary Division, Department of Internal Medicine and Hepatitis Center, Kaohsiung Medical University Hospital, Kaohsiung Medical University, Kaohsiung, Taiwan

${ }^{3}$ Division of Gastroenterology and Hepatology, Mayo Clinic, Rochester, MN, USA

${ }^{4}$ Division of Gastroenterology, University of Michigan Health System, Ann Arbor, MI, USA

${ }^{5}$ Stanford Prevention Research Center,

Stanford Cancer Institute, Stanford, CA, USA

Correspondence

Mindie H. Nguyen, MD, MAS, Associate Professor of Medicine, Division of Gastroenterology and Hepatology, Stanford University Medical Center, Palo Alto, CA, USA. Email: mindiehn@stanford.edu

Handling Editor: Janus Ong

\begin{abstract}
Background \& Aims: Although hepatitis B virus (HBV) and hepatitis C virus (HCV) infections remain major risk factors for hepatocellular carcinoma (HCC), non-viral causes of HCC, particularly non-alcoholic fatty liver disease (NAFLD), are becoming increasingly prevalent. The aim of this study was to compare the clinical characteristics and survival of cryptogenic and viral HCC.

Methods: We conducted a retrospective cohort study involving 3878 consecutive HCC patients seen at two tertiary centres in the United States and one in Taiwan from 2004 to 2014. We compared the clinical characteristics, treatment and survival of patients by underlying aetiology: cryptogenic $(n=696)$, HBV $(n=1304)$ or HCV ( $n=1878)$.

Results: Cirrhosis was present in $66.8 \%$ of the cryptogenic HCC patients, compared with $74.7 \%$ of HBV-related HCC (HBV-HCC) $(P=.001)$ and $85.9 \%$ of HCV-HCC $(P<.001)$. Compared to viral HCC, cryptogenic HCC patients presented with larger tumours and at later stages of disease. Five-year overall survival was $16.3 \%$ among cryptogenic HCC patients compared with $31.9 \%$ among HBV-HCC patients and $27.7 \%$ among HCV-HCC patients ( $P<.001$ for both by the log-rank test). HCC aetiology was not an independent predictor of survival, though ethnicity, cirrhosis status, meeting Milan criteria and treatment allocation were.

Conclusions: Compared with viral HCC patients, those with cryptogenic HCC had lower prevalence of cirrhosis, were diagnosed with larger tumours at more advanced stages of disease, and had poorer overall survival. Additional efforts are needed to identify patients at risk of cryptogenic HCC and to identify cryptogenic HCC at earlier stages of disease.
\end{abstract}

KEYWORDS

cryptogenic HCC, hepatitis B, hepatitis C, hepatocellular carcinoma

Abbreviations: AFP, alpha-foetoprotein; ALT, alanine transaminase; AST, aspartate aminotransferase; BCLC stage, Barcelona clinic liver cancer stage; BMI, body mass index; CAD, coronary artery disease; CT, computed tomography; HBV-HCC, HBV-related HCC; HBV, hepatitis B virus; HCC, hepatocellular carcinoma; HCV-HCC, HCV-related HCC; HCV, hepatitis C virus; HR, hazard ratio; IQR, interquartile range; MELD, model for end-stage liver disease; MRI, magnetic resonance imaging; NAFLD-HCC, NAFLD-related HCC; NAFLD, non-alcoholic fatty liver disease; NASH, non-alcoholic steatohepatitis; RE, radioembolization; RFA, radiofrequency ablation; TACE, transcatheter arterial chemoembolization. 


\section{1 | INTRODUCTION}

Hepatocellular carcinoma (HCC) is a major cause of cancer mortality worldwide and was the fourth leading cause of death with 800000 deaths in $2015 .{ }^{1}$ In the United States and Taiwan, where we practice, 5 -year survival for liver cancer is $18 \%$ and $28.9 \%$ respectively. ${ }^{2,3}$

While chronic infections with hepatitis B virus (HBV) and hepatitis $\mathrm{C}$ virus (HCV) are the major HCC risk factors globally (53\% and $25 \%$, respectively), other chronic liver diseases are also associated with HCC, such as non-alcoholic fatty liver disease (NAFLD). ${ }^{4}$ NAFLD is an increasingly important cause of $\mathrm{HCC}$ with an estimated global prevalence of $25 \%$ and rising. ${ }^{5-8}$

Current HCC surveillance guidelines focus on HCC in the setting of chronic viral hepatitis or cirrhosis. ${ }^{9}$ However, a growing body of evidence suggests that a third or more of NAFLD-related HCC develops in patients without a known history of cirrhosis. ${ }^{10-15}$ Some studies have also found that patients with non-viral aetiologies of HCC are diagnosed at more advanced stages, possibly because of lower rates of surveillance. ${ }^{14-16}$ More data are needed to understand the epidemiology of HCC associated with non-viral aetiologies, particularly NAFLD, in order to inform guidelines moving forward.

A clear-cut diagnosis of NAFLD or its inflammatory counterpart, non-alcoholic steatohepatitis (NASH), is not always possible at the time of HCC diagnosis. Over time, hepatic steatosis may be replaced by fibrosis and cirrhosis, and the metabolic derangements associated with NAFLD, such as obesity, may not be apparent in end-stage liver disease. ${ }^{17}$ As such, there is increasing acknowledgement that a significant proportion of cryptogenic HCC-that is, HCC in the absence of chronic viral infection, alcohol use or other diagnosed liver disease-is likely because of NAFLD. ${ }^{5,18-20}$

To augment the body of knowledge on cryptogenic HCC, we conducted a retrospective cohort study of 3878 consecutive HCC patients diagnosed between 2004 and 2014 in the United States and Taiwan comparing the clinical characteristics and survival of viral-related HCC against those of cryptogenic HCC.

\section{2 | PATIENTS AND METHODS}

\section{1 | Study design and patient population}

This retrospective cohort study involved 3878 consecutive cases of HBV-related, HCV-related or cryptogenic HCC seen at two tertiary hospitals in the United States and one in Taiwan between 2004 and 2014. HCC diagnosis was based on histology, cytology, or non-invasive criteria recommended by the American Association for the Study of Liver Diseases (AASLD). ${ }^{9}$ The study protocol was approved by the institutional review boards of the Stanford University Medical Center, the Mayo Clinic in Rochester, and Kaohsiung Medical University Hospital. An exemption from informed consent was granted because of the minimal risk posed to participants in this chart review study.

Adults aged 18 or older were eligible for inclusion if they had $\mathrm{HCC}$ and an underlying diagnosis of $\mathrm{HBV}, \mathrm{HCV}$, or if their $\mathrm{HCC}$ was cryptogenic. Diagnoses of HBV and HCV were based on serological

\section{Key points}

- One-third of cryptogenic hepatocellular carcinoma (HCC) patients were non-cirrhotic, significantly more than viral HCC patients.

- Cryptogenic HCC patients presented with larger tumours and at more advanced stages of disease than viral HCC patients.

- Compared to viral HCC patients, those with cryptogenic HCC had worse overall survival despite often receiving treatments with curative intent.

- Cryptogenic aetiology of HCC was not an independent predictor of survival after adjusting for factors such as stage of disease and treatment strategy.

testing as well as nucleic acid tests for viraemia. Cryptogenic HCC was defined as HCC in the absence of any history of regular alcohol use and without a confirmed chronic liver disease such as chronic hepatitis B or C, autoimmune or metabolic liver disease such as primary biliary cirrhosis, primary sclerosing cholangitis, haemochromatosis or Wilson's disease. Patients with HCC in the presence of multiple underlying liver diseases (eg, $\mathrm{HBV}$ and $\mathrm{HCV}$ co-infection) were excluded. Alcohol intake was not routinely quantified, but cases of HCC deemed to be alcohol-related by the examining physicians (as documented in their clinical notes) were excluded. Patient inclusion and exclusion are illustrated in Figure S1.

\subsection{Definition of cirrhosis}

Cirrhosis status was determined based on histology, imaging and chart review. Patients were considered to have cirrhosis if they had F4 fibrosis on histology, if they had clinical evidence of portal hypertension (platelets $<120000 / \mu \mathrm{L}$, splenomegaly, ascites or gastroesophageal varices on imaging) or if they had hepatic decompensation (hepatic encephalopathy, ascites, variceal bleeding) within 6 months of HCC diagnosis.

\section{3 | Tumour staging and survival outcomes}

Tumour stage was assessed by the Milan criteria for transplant and the Barcelona clinic liver cancer (BCLC) staging system. Tumour size and other imaging characteristics were derived from computed tomography (CT) or magnetic resonance imaging (MRI).

Survival data were based on the date of HCC diagnosis and the date of death or last follow-up date.

\section{4 | Statistical analysis}

Descriptive statistics of categorical variables were reported as proportions (\%), while continuous variables were reported as means with standard deviations or medians with interquartile ranges. Comparisons 
of descriptive statistics were made using the Student's $t$ test, the chisquare test, or the Mann-Whitney $U$ test for normally distributed continuous variables, categorical variables, and non-normally distributed continuous variables respectively.

Five-year overall survival was the primary outcome. The primary predictor variable was HCC aetiology (HBV, HCV or cryptogenic). Secondary predictors included ethnicity, cirrhosis, tumour stage and treatment strategy. Treatments such as liver transplantation, surgical resection and radiofrequency ablation (RFA) were considered treatments with curative intent, while treatments such as transcatheter arterial chemoembolization (TACE), radioembolization (RE) and sorafenib were considered palliative. Univariate and multivariate survival models were constructed using Cox proportional hazards models. Relevant variables that were significant (defined as association with $P<.05)$ in the univariate analysis were included in the multivariate model. Kaplan-Meier survival curves and 5-year survival rates for independent subgroups were compared using the log-rank test.

All statistical analyses were performed in Stata, version 14 (Stata Corporation, College Station, TX, USA). Statistical significance was defined as a two-tailed $P$ value of $<.05$.

\section{3 | RESULTS}

\section{1 | Baseline patient clinical characteristics}

Of the 3878 HCC patients, 696 (18.0\%) were cryptogenic, 1304 (33.6\%) were HBV-related and 1878 (48.4\%) were HCV-related. The median date of HCC diagnosis was 2008.5 for cryptogenic HCC patients, 2008 for HBV-related HCC (HBV-HCC) patients, and 2009 for
HCV-HCC patients. Baseline clinical and laboratory characteristics of the patients by HCC aetiology are shown in Tables 1 and 2. Compared to patients with HBV-HCC or HCV-HCC, those with cryptogenic $\mathrm{HCC}$ were older, had higher body mass index (BMI)s, and were more likely to have metabolic comorbidities such as obesity, diabetes and hypertension.

Clinically apparent cirrhosis was less common among cryptogenic HCC patients; $66.8 \%$ of cryptogenic patients had cirrhosis compared to $74.7 \%$ of HBV-HCC patients $(P=.001)$ and $85.9 \%$ of HCV-HCC patients $(P<.001)$

\section{2 | Tumour characteristics}

Table 3 compares tumour characteristics across the three aetiologies. Patients with cryptogenic HCC had larger tumours and more advanced disease at presentation than patients with HBV-HCC or HCV-HCC. The median maximum tumour size of cryptogenic HCC patients was $6.0 \mathrm{~cm}$ at diagnosis compared to $3.9 \mathrm{~cm}$ for $\mathrm{HBV}-\mathrm{HCC}$ and $3.2 \mathrm{~cm}$ for HCV-HCC ( $P<.001$ for both comparisons). Cryptogenic HCC patients were more likely to have extrahepatic metastases (16.2\%) compared to HBV-HCC (11.2\%, $P=.002)$ and HCV-HCC $(6.7 \%, P<.001)$. Less than one-third (28.2\%) of cryptogenic HCC patients met Milan criteria for transplantation compared to nearly half of HBV-HCC patients (45.4\%) and $55.8 \%$ of HCV-HCC patients ( $P<.001$ for both comparisons).

\section{3 | Treatment allocation}

Despite having more advanced tumours at presentation, cryptogenic HCC patients were significantly more likely to receive

TAB LE 1 Baseline patient clinical characteristics, by hepatocellular carcinoma (HCC) aetiology

\begin{tabular}{|c|c|c|c|c|c|c|}
\hline & Cryptogenic ( $\mathrm{N}=696$ ) & $\mathrm{HBV}(\mathrm{N}=1304)$ & $P$ value & $\mathrm{HCV}(\mathrm{N}=1878)$ & $P$ value & $\begin{array}{l}\text { Overall } \\
(\mathrm{N}=3878)\end{array}$ \\
\hline $\operatorname{Age}^{a}(y)$ & $67.2 \pm 13.4$ & $58.3 \pm 12.2$ & $<.001$ & $63.0 \pm 9.9$ & $<.001$ & $62.2 \pm 11.8$ \\
\hline Male & $440(63.2 \%)$ & 1074 (82.4\%) & $<.001$ & $1248(66.5 \%)$ & .125 & $2762(71.2 \%)$ \\
\hline Asian & $317(45.6 \%)$ & $1251(95.9 \%)$ & $<.001$ & $1040(55.4 \%)$ & $<.001$ & $2608(67.3 \%)$ \\
\hline US site & $412(59.2 \%)$ & $349(26.8 \%)$ & $<.001$ & $1054(56.1 \%)$ & .162 & $1815(46.8 \%)$ \\
\hline History of regular alcohol use & $0(0 \%)$ & $380(29.3 \%)$ & $<.001$ & $801(43.0 \%)$ & $<.001$ & $1181(30.7 \%)$ \\
\hline Body mass index ${ }^{a}\left(\mathrm{~kg} / \mathrm{m}^{2}\right)$ & $27.7 \pm 6.2$ & $24.4 \pm 4.0$ & $<.001$ & $26.0 \pm 5.3$ & $<.001$ & $25.8 \pm 5.2$ \\
\hline Hypertension (HTN) & $354(58.2 \%)$ & $403(31.7 \%)$ & $<.001$ & 855 (47.1\%) & $<.001$ & $1612(43.6 \%)$ \\
\hline Diabetes (DM) & $278(45.7 \%)$ & $299(23.5 \%)$ & $<.001$ & $594(32.8 \%)$ & $<.001$ & $1171(31.8 \%)$ \\
\hline$\geq 2$ of obesity ${ }^{b}$, HTN, DM & $297(44.8 \%)$ & $295(22.9 \%)$ & $<.001$ & $604(32.5 \%)$ & $<.001$ & $1196(31.4 \%)$ \\
\hline Coronary artery disease & $130(21.5 \%)$ & $40(3.1 \%)$ & $<.001$ & $108(6.0 \%)$ & $<.001$ & $278(7.6 \%)$ \\
\hline Symptomatic at diagnosis & 395 (58.0\%) & $491(39.1 \%)$ & $<.001$ & $670(42.8 \%)$ & $<.001$ & $1556(44.4 \%)$ \\
\hline Cirrhosis & $338(66.8 \%)$ & 919 (74.7\%) & .001 & 1497 (85.9\%) & $<.001$ & $2754(79.2 \%)$ \\
\hline Ascites & 198 (30.8\%) & $345(27.2 \%)$ & .099 & $442(24.3 \%)$ & .001 & $985(26.4 \%)$ \\
\hline Encephalopathy & $56(8.4 \%)$ & $79(6.2 \%)$ & .063 & $152(8.3 \%)$ & .92 & $287(7.6 \%)$ \\
\hline
\end{tabular}

CAD, coronary artery disease; HBV, hepatitis B virus; $\mathrm{HCV}$, hepatitis $\mathrm{C}$ virus.

$P$ values are for the comparison to cryptogenic.

${ }^{a}$ Reported as mean \pm standard deviation.

${ }^{b}$ Obesity defined as $\mathrm{BMI} \geq 30$ for non-Asians and $\geq 25$ for Asians (both East Asian and South Asian). 
TAB LE 2 Baseline patient laboratory characteristics, by hepatocellular carcinoma (HCC) aetiology

\begin{tabular}{|c|c|c|c|c|c|c|}
\hline & Cryptogenic $(\mathrm{N}=696)$ & $\mathrm{HBV}(\mathrm{N}=1304)$ & $P$ value & $\mathrm{HCV}(\mathrm{N}=1878)$ & $P$ value & Overall $(\mathrm{N}=3878)$ \\
\hline Platelet count ${ }^{\mathrm{b}}(\mathrm{K} / \mu \mathrm{L})$ & 183.5 (IQR 121-259) & 152 (IQR 104-217) & $<.001$ & 114 (IQR 76-163) & $<.001$ & 137 (IQR 89-197) \\
\hline Total bilirubin ${ }^{\mathrm{b}}$ (mg/dL) & 0.9 (IQR 0.6-1.5) & 1 (IQR 0.7-1.5) & .003 & 1.1 (IQR 0.7-1.7) & $<.001$ & 1 (IQR 0.7-1.6) \\
\hline Albumin $^{a}(g / d L)$ & $3.5 \pm 0.6$ & $3.5 \pm 0.6$ & .323 & $3.3 \pm 0.6$ & $<.001$ & $3.4 \pm 0.6$ \\
\hline $\begin{array}{l}\text { International normalized } \\
\text { ratio }^{\text {b }}\end{array}$ & 1.1 (IQR 1-1.2) & 1.1 (IQR 1-1.2) & $<.001$ & 1.1 (IQR 1-1.3) & $<.001$ & 1.1 (IQR 1-1.2) \\
\hline $\begin{array}{l}\text { Aspartate transaminase }{ }^{b} \\
(\mathrm{U} / \mathrm{L})\end{array}$ & 55 (IQR 36-95) & 59 (IQR 38-108) & .068 & 80.5 (IQR 52-124) & $<.001$ & 69 (IQR 43-115) \\
\hline $\begin{array}{l}\text { Alanine transaminase }{ }^{b} \\
(\mathrm{U} / \mathrm{L})\end{array}$ & 42 (IQR 27-61) & 48 (IQR 34-75) & $<.001$ & 67 (IQR 40-107) & $<.001$ & 69 (IQR 43-115) \\
\hline $\log _{10} A_{F P}^{b}(n g / d L)$ & 3.3 (IQR 1.6-7.2) & 4.3 (IQR 2.3-6.9) & $<.001$ & 3.7 (IQR 2.3-6) & .008 & 3.8 (IQR 2.2-6.4) \\
\hline CTP $A^{c}$ & $124(47.7 \%)$ & $508(62.9 \%)$ & $<.001$ & 719 (56.0\%) & .027 & $1351(57.5 \%)$ \\
\hline СТP B & $113(43.5 \%)$ & $247(30.6 \%)$ & & 488 (38.1\%) & & 848 (36.1\%) \\
\hline CTP C & 23 (8.9\%) & $53(6.6 \%)$ & & $76(5.9 \%)$ & & $152(6.5 \%)$ \\
\hline MELD $^{b}$ & 9 (IQR 7-12) & 9 (IQR 7-11) & .787 & 9 (IQR 8-13) & .001 & 9 (IQR 7-12) \\
\hline
\end{tabular}

AFP, alpha-foetoprotein; HBV, hepatitis B virus; HCV, hepatitis C virus; MELD, model for end-stage liver disease.

$P$ values are for the comparison to cryptogenic.

${ }^{\mathrm{a}}$ Reported as mean \pm standard deviation.

${ }^{\mathrm{b}}$ Reported as median with interquartile range.

${ }^{\mathrm{c} C h i l d-T u r c o t t e}$ Pugh class calculated for cirrhotic patients only.

TABLE 3 Tumour characteristics, by hepatocellular carcinoma (HCC) aetiology

\begin{tabular}{|lllllrl} 
& $\begin{array}{l}\text { Cryptogenic } \\
(\mathrm{N}=696)\end{array}$ & HBV (N = 1304) & P value & HCV (N = 1878) & P value & Overall (N = 3878) \\
\hline Max. tumour size (cm) & $6.0(\mathrm{IQR} 3.4-9.7)$ & $3.9(\mathrm{IQR} 2.4-7.3)$ & $<.001$ & $3.2(\mathrm{IQR} 2.1-5.2)$ & $<.001$ & $3.7(\mathrm{IQR} 2.3-6.5)$ \\
\hline Multifocal & $276(44.5 \%)$ & $342(33.5 \%)$ & $<.001$ & $602(35.0 \%)$ & $<.001$ & $1220(36.3 \%)$ \\
\hline Vascular invasion & $127(19.7 \%)$ & $203(17.9 \%)$ & .347 & $232(13.0 \%)$ & $<.001$ & $562(15.8 \%)$ \\
\hline $\begin{array}{l}\text { Extrahepatic } \\
\text { metastasis }\end{array}$ & $107(16.2 \%)$ & $140(11.2 \%)$ & .002 & $123(6.7 \%)$ & $<.001$ & $370(9.9 \%)$ \\
\hline Within Milan criteria & $187(28.2 \%)$ & $530(45.4 \%)$ & $<.001$ & $974(55.8 \%)$ & $<.001$ & $1691(47.3 \%)$ \\
\hline BCLC C/D & $214(46.8 \%)$ & $323(35.5 \%)$ & $<.001$ & $363(26.4 \%)$ & $<.001$ & $900(32.8 \%)$ \\
\hline
\end{tabular}

BCLC, Barcelona clinic liver cancer; HBV, hepatitis B virus; $\mathrm{HCV}$, hepatitis $\mathrm{C}$ virus.

$P$ values are for the comparison to cryptogenic.

treatments with curative intent compared to patients with viral aetiologies $(31.5 \%$ for cryptogenic HCC, $23.0 \%$ for HBV-HCC; $P<.001$, and $26.0 \%$ for HCV-HCC; $P=.011$ ) (Table 4). Resection in particular was more common among cryptogenic HCC patients (26.6\%) than in HBV-HCC $(16.5 \%, P<.001)$ or HCV-HCC patients $(11.2 \%, P<.001)$.

\section{4 | Overall survival}

Average length of follow-up was 1.63 years (SD: 1.97 years). The average length of follow-up by aetiology was 1.1 years (SD: 1.51 years) for the cryptogenic group, 1.6 years (SD: 2.07 years) for the HBV group and 1.8 years (SD: 2.00 years) for the HCV group. The rate of loss to follow-up at 5 years was not significantly different between the cryptogenic HCC group and either of the viral HCC groups $(47.0 \%$ cryptogenic, $51.7 \%$ HBV, $46.8 \%$ HCV; crypto vs HBV $P=.06$, crypto vs HCV $P=.92)$.

Five-year overall survival was worse among cryptogenic $\mathrm{HCC}$ patients (16.3\%) compared with either HBV-HCC $(31.9 \%, P<.001)$ or HCV-HCC patients $(27.7 \%, P<.001)$ (Figure $1 \mathrm{~A})$. This result persisted after stratification by cirrhosis (Figure 1B,C). Cirrhotic cryptogenic HCC patients had worse 5 -year survival than cirrhotic viral HCC (19.4\% vs $26.5 \%, P<.001)$. Similarly, non-cirrhotic cryptogenic HCC patients had worse 5-year survival than non-cirrhotic viral HCC $(28.2 \%$ vs $47.7 \%, P<.001)$.

Since cryptogenic HCC patients underwent curative treatments at a higher rate than viral HCC patients, we also examined survival by aetiology for patients receiving either surgical resection or RFA as their primary HCC therapy. Cryptogenic HCC patients undergoing either resection or RFA still had worse 5-year overall survival (38.1\%) than either HBV-HCC (67.3\%, $P<.001)$ or HCV-HCC (45.2\%, $P=.02)$ (Figure 1D). 
TAB LE 4 Treatment allocation, by hepatocellular carcinoma (HCC) aetiology

\begin{tabular}{|c|c|c|c|c|c|c|}
\hline & $\begin{array}{l}\text { Cryptogenic } \\
(N=696)\end{array}$ & $\operatorname{HBV}(\mathrm{N}=1304)$ & $P$ value & $\mathrm{HCV}(\mathrm{N}=1878)$ & $P$ value & $\begin{array}{l}\text { Overall } \\
(\mathrm{N}=3878)\end{array}$ \\
\hline Transplant & $13(3.2 \%)$ & 21 (1.7\%) & .062 & 132 (8.3\%) & $<.001$ & $166(5.1 \%)$ \\
\hline RFA & 39 (9.3\%) & $76(6.2 \%)$ & .029 & 154 (9.5\%) & .888 & 269 (8.2\%) \\
\hline TACE & 252 (54.6\%) & $651(52.2 \%)$ & .381 & 1039 (61.1\%) & .011 & $1942(56.9 \%)$ \\
\hline Sorafenib & $11(2.8 \%)$ & $26(2.1 \%)$ & .447 & $37(2.3 \%)$ & .621 & 74 (2.3\%) \\
\hline Curative intent & 174 (31.5\%) & $291(23.0 \%)$ & $<.001$ & $448(26.0 \%)$ & .011 & $913(25.8 \%)$ \\
\hline \multicolumn{7}{|l|}{ Primary treatment } \\
\hline Transplant & $13(2.4 \%)$ & 21 (1.7\%) & \multirow[t]{2}{*}{.005} & $132(7.7 \%)$ & \multirow[t]{2}{*}{$<.001$} & $166(4.7 \%)$ \\
\hline Resection/RFA & $161(29.1 \%)$ & $270(21.4 \%)$ & & $316(18.3 \%)$ & & $747(21.1 \%)$ \\
\hline
\end{tabular}

Curative intent: Transplant, resection or radiofrequency ablation. HBV, hepatitis B virus; HCV, hepatitis C virus; RE, radioembolization; RFA, radiofrequency ablation; TACE, transcatheter arterial chemoembolization.

$P$ values are for the comparison to cryptogenic.

\section{5 | Predictors of survival}

Favourable predictors of 5-year survival in univariate Cox proportional hazard models included female gender, younger age, Asian or Hispanic ethnicity (compared to Caucasian ethnicity), absence of cirrhosis, absence of coronary artery disease (CAD), meeting Milan criteria, curative or palliative treatments (compared to no treatment) and viral aetiology (Table 5). In the multivariate analysis, viral aetiology was no longer a significant predictor of survival. Significant independent predictors of survival were Asian or Hispanic ethnicity, absence of cirrhosis, lower model for end-stage liver disease (MELD) score, meeting Milan criteria and curative or palliative treatments.

\subsection{Analysis by United States vs Taiwan sites}

The distribution of aetiologies and treatment strategies differed between the US and Taiwan sites. The majority of the cryptogenic and HCV-HCC patients were from the United States whereas the majority of the HBV-HCC patients were from Taiwan. The US sites were more likely to perform curative treatments such as transplant (13.9\% vs $0.1 \%, P<.001)$, resection (19.6\% vs $12.7 \%, P<.001)$ and RFA (11.3\% vs 6.4\%, $P<.001)$ (Table S1). However, cryptogenic $\mathrm{HCC}$ patients had worse survival than viral HCC patients at both US and Taiwan sites. At the US sites, 5-year overall survival was $16.6 \%$ for the cryptogenic HCC patients compared to $39.8 \%$ and $27.9 \%$ for the HBV-HCC and HCV-HCC patients respectively $(P<.001$ for both) (Figure S2A). At the Taiwan site, 5 -year overall survival was $15.6 \%$ for the cryptogenic HCC patients, $27.5 \%$ for the HBV-HCC patients $(P=.03)$ and $26.2 \%$ for the HCV-HCC patients $(P<.001)$ (Figure S2B).

\subsection{Hepatocellular carcinoma surveillance}

Data on HCC surveillance were available for 984 patients from Stanford University Medical Center and the Mayo Clinic. Surveillance was defined as US or triphasic CT imaging of the liver at 6 month intervals prior to the diagnosis of HCC. Surveillance status was determined through manual chart review.

Of these, 330 had cryptogenic HCC, 160 had HBV-HCC and 494 had HCV-HCC. HCV-HCC patients had a significantly higher rate of surveillance than cryptogenic HCC patients (38.3\% vs $18.8 \%$, $P=.001$ ). There was a trend towards a higher rate of surveillance among the HBV-HCC patients compared with the cryptogenic HCC patients ( $26.3 \%$ vs $18.8 \%, P=.058)$

Among those under surveillance, there were no significant differences in tumour size or stage (based on metastases and Milan criteria) across aetiologies (Table S2). Among those not under surveillance, the cryptogenic group had larger and more advanced tumours than either the HBV or HCV groups (Table S3).

Patients under surveillance had a 5 -year survival of $22.5 \%$ compared to $17.4 \%$ for those not under surveillance $(P<.001)$ (Figure S3). HCC surveillance was a positive predictor of survival in univariate analysis (Table 5). However, surveillance was not included in the multivariate model because of the lack of data from all sites.

\section{4 | DISCUSSION}

In this large study of $3878 \mathrm{HCC}$ patients, we found that relative to patients with HBV- or HCV-HCC, patients with cryptogenic HCC were less likely to have cirrhosis, had larger tumours, had more advanced disease, and had worse 5 -year overall survival. However, HCC 
(A)
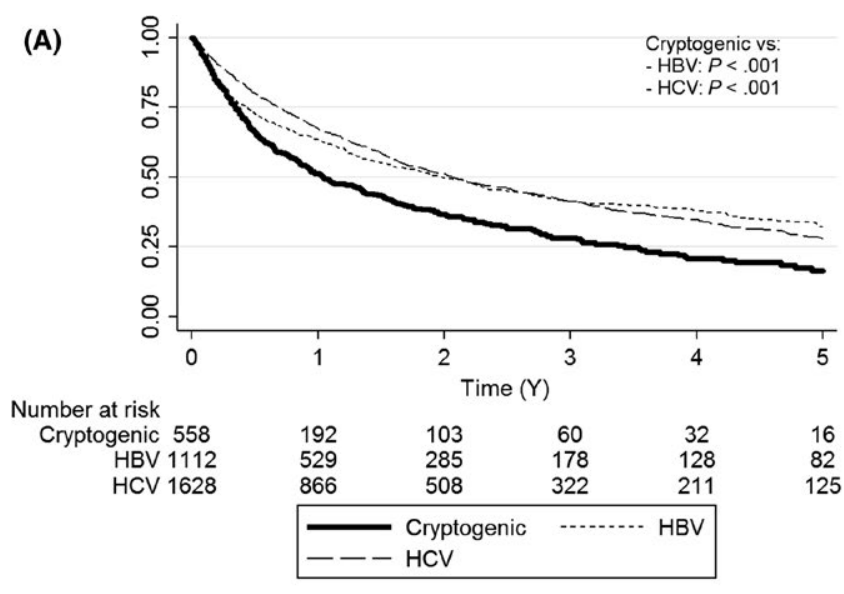

(C)

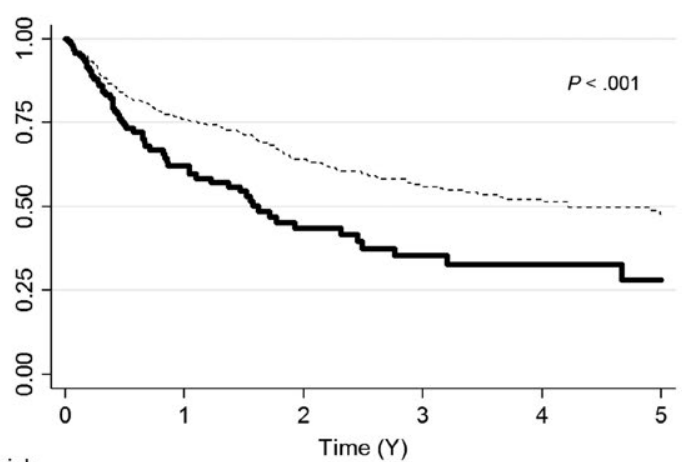

Number at risk Non-cirrhotic crypto 123 Non-cirrhotic viral 455

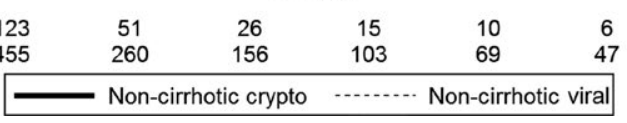

(B)

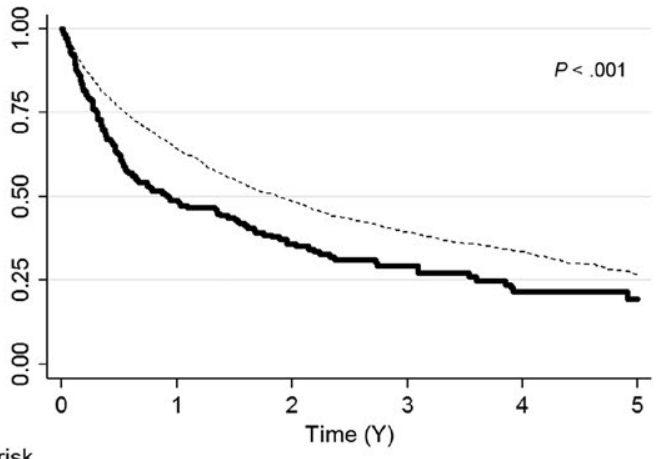

Number at risk $\begin{array}{llllll}\text { Cirrhotic cryptogenic } 280 & 91 & 51 & 30 & 17 & 9\end{array}$ \begin{tabular}{llllll|} 
Cirrhotic viral2135 & 1076 & 608 & 385 & 262 & 156 \\
\hline & & Cirrhotic & cryptogenic & .......... & Cirrhotic viral
\end{tabular}

(D)

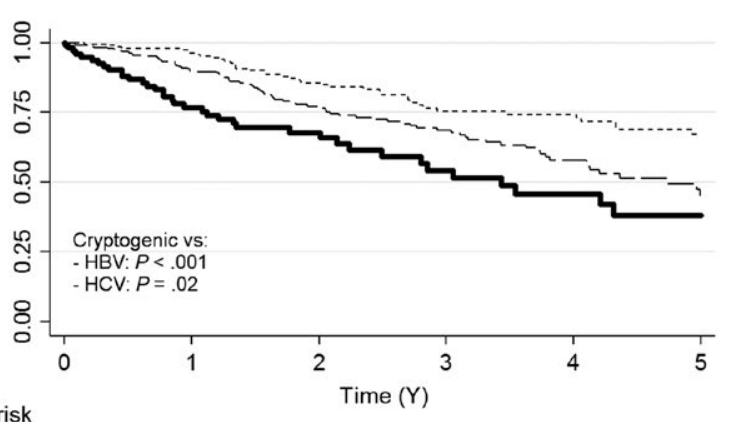

Number at risk

Cryptogenic 109

HBV 200

HCV 251

FIGURE 1 Five-y overall survival for patients with cryptogenic and viral hepatocellular carcinoma (HCC). A, Overall survival, by HCC aetiology. B, Overall survival for cirrhotic patients only, by HCC aetiology. C, Overall survival for non-cirrhotic patients only, by HCC aetiology. $\mathrm{D}$, Overall survival for patients undergoing resection or radiofrequency ablation (RFA) as their primary treatment, by HCC aetiology

aetiology was not an independent predictor of survival after adjusting for covariates such as ethnicity, cirrhosis status, tumour stage and treatment strategy.

It should be noted that we found worse survival in the cryptogenic $\mathrm{HCC}$ group despite that group being more likely to receive treatments with curative intent, particularly surgical resection. It is possible that resection is more commonly offered to this group of patients because cirrhosis is less prevalent compared to patients with viral HCC. However, cryptogenic HCC patients receiving resection or RFA still had worse survival compared to viral $\mathrm{HCC}$ patients receiving the same treatments. The advanced stage of cryptogenic $\mathrm{HCC}$ at presentation is likely an important contributor to this discrepancy. Cryptogenic HCC patients may already have occult metastases at presentation, or may require larger sections of liver to be resected or ablated. These patients also had more comorbidities which could reduce overall survival, such as CAD and diabetes, though neither of these were independent predictors of survival in our model.

In a subset analysis of patients for whom we had HCC surveillance data, we found that the cryptogenic group had lower rates of surveillance than either of the viral groups. Those who had prior HCC surveillance had better survival than those who did not. Among the patients under surveillance, there were no significant differences in tumour size or stage across the three aetiologies. These findings suggest that a lack of adequate surveillance contributed to the differences between the cryptogenic and viral groups in tumour stage and survival.

There may have been several barriers to adequate HCC surveillance in cryptogenic HCC patients. Firstly, one-third of cryptogenic $\mathrm{HCC}$ patients in our cohort did not have cirrhosis and hence would not have met current criteria for HCC surveillance. Secondly, prior studies have reported lower HCC surveillance rates for NAFLD-related cirrhosis compared to other forms of cirrhosis, perhaps because of lack of awareness about the risk of NAFLD progressing to HCC. ${ }^{14-16}$ Thirdly, the sensitivity of ultrasound surveillance may be limited in the NAFLD/cryptogenic HCC population. Obesity and NAFLD cirrhosis have both been associated with inadequacy of ultrasound for the detection of hepatic tumours. ${ }^{21,22}$ These latter two factors may have contributed to our finding that even among cirrhotic patients, cryptogenic HCC patients had worse survival. Increasing provider awareness of the risk of HCC in NAFLD may improve early detection, and more work is needed to determine whether or how CT and MRI screening should be incorporated into HCC surveillance strategies for patients with NAFLD or obesity.

The strengths of our study include its large size and diverse patient population. To our knowledge, this is the largest international cohort 
TABLE 5 Predictors of 5-y mortality

\begin{tabular}{|c|c|c|c|c|}
\hline \multirow[b]{2}{*}{ Predictor } & \multicolumn{2}{|l|}{ Univariate } & \multicolumn{2}{|l|}{ Multivariate } \\
\hline & $\mathrm{HR}(95 \% \mathrm{Cl})$ & $P$ value & $\mathrm{HR}(95 \% \mathrm{Cl})$ & $P$ value \\
\hline Male & $1.14(1.02-1.28)$ & .017 & $0.96(0.83-1.11)$ & .593 \\
\hline Age & $1.01(1.00-1.01)$ & .019 & $1.00(1.00-1.01)$ & .492 \\
\hline \multicolumn{5}{|l|}{ Ethnicity $^{a}$} \\
\hline Caucasian & 1 & Reference & 1 & Reference \\
\hline Asian (Taiwan) & $0.89(0.79-1.00)$ & .049 & $0.82(0.68-0.99)$ & .039 \\
\hline Asian (US) & $0.59(0.50-0.69)$ & $<.001$ & $0.51(0.40-0.64)$ & $<.001$ \\
\hline African-American & $1.06(0.74-1.52)$ & .733 & $0.89(0.52-1.51)$ & .657 \\
\hline Hispanic & $0.69(0.56-0.86)$ & .001 & $0.59(0.45-0.77)$ & $<.001$ \\
\hline Cirrhosis & 1.55 (1.34-1.79) & $<.001$ & $1.41(1.19-1.68)$ & $<.001$ \\
\hline Diabetes & $1.02(0.92-1.14)$ & .687 & & \\
\hline $\begin{array}{c}\text { Coronary artery } \\
\text { disease (CAD) }\end{array}$ & $1.37(1.15-1.63)$ & $<.001$ & $1.17(0.87-1.59)$ & .300 \\
\hline MELD & $1.05(1.04-1.06)$ & $<.001$ & $1.05(1.04-1.06)$ & $<.001$ \\
\hline $\begin{array}{l}\text { Within Milan } \\
\text { criteria }\end{array}$ & $0.30(0.27-0.34)$ & $<.001$ & $0.33(0.29-0.38)$ & $<.001$ \\
\hline HCC Surveillance & $0.54(0.42-0.70)$ & $<.001$ & & \\
\hline \multicolumn{5}{|l|}{ Primary treatment } \\
\hline No treatment & 1 & Reference & 1 & Reference \\
\hline Curative & $0.09(0.08-0.11)$ & $<.001$ & $0.10(0.08-0.12)$ & $<.001$ \\
\hline Palliative & $0.33(0.29-0.36)$ & $<.001$ & $0.35(0.30-0.40)$ & $<.001$ \\
\hline \multicolumn{5}{|l|}{ Aetiology } \\
\hline Cryptogenic & 1 & Reference & 1 & Reference \\
\hline HBV & $0.69(0.60-0.80)$ & $<.001$ & $0.99(0.80-1.22)$ & .901 \\
\hline $\mathrm{HCV}$ & $0.66(0.58-0.75)$ & $<.001$ & $0.83(0.68-1.02)$ & .083 \\
\hline
\end{tabular}

HBV, hepatitis B virus; HCC, hepatocellular carcinoma; HCV, hepatitis C virus; MELD, model for endstage liver disease.

${ }^{a}$ Caucasian, $\mathrm{N}=752$; Asian (Taiwan), $\mathrm{N}=1655$; Asian (US), $\mathrm{N}=510$; African-Am., $\mathrm{N}=$ 54; Hispanic, $\mathrm{N}=208$.

that has been assembled to compare viral and non-viral HCC. Our data are also consistent with previous studies which found that NAFLD$\mathrm{HCC}$ often arises in patients without clinically apparent cirrhosis and that NAFLD-HCC tends to present with larger tumours and at later stages. ${ }^{10-16,23-25}$ A nationwide survey in Japan found cirrhosis in $62 \%$ of NAFLD-HCC cases, while a US Department of Veterans Affairs study found cirrhosis in $58.3 \%$ of NAFLD-HCC patients; we found cirrhosis in $66.8 \%$ of cryptogenic HCC patients in our study, consistent with these prior reports. ${ }^{13,14}$

Ours is also the largest study, thus far, to evaluate survival in the cryptogenic HCC population. Survival data from prior studies have been mixed, though the larger studies generally have had results similar to ours. ${ }^{14,15,25,26}$ One study from Taiwan involving 366 cryptogenic $\mathrm{HCC}$ patients found worse long-term overall survival in the cryptogenic group compared to the viral/alcoholic HCC group; this difference was no longer significant after controlling for confounding variables. ${ }^{25} \mathrm{An}$ Italian study involving 145 NAFLD-HCC patients found a similar pattern: worse overall survival in the uncorrected analysis and similar survival when controlling for covariates. ${ }^{15}$ The aforementioned Veterans
Affairs study by Mittal et al included 120 NAFLD-HCC patients and did not find any difference in 1-year survival compared to alcohol or HCV-HCC. ${ }^{14}$ Their results may differ from ours because of a shorter follow-up period and factors specific to the veteran population.

There are a number of limitations to our study. Firstly, the study is retrospective in design, though our primary outcome is overall survival, an objective and clear outcome. Our cohort is also drawn from tertiary referral centres and may not be representative of the wider population of HCC patients. However, our cohort is geographically diverse. For most patients in our cohort, cirrhosis was diagnosed based on imaging, laboratory values or clinical history rather than liver histology. These criteria are not sensitive for subclinical cirrhosis and may underestimate the prevalence of cirrhosis in our cohort. We are also limited to discussing cryptogenic HCC rather than NAFLD-HCC. We cannot reliably obtain formal diagnoses of NAFLD-HCC from our data despite individual chart review, as hepatic steatosis is not reliably present in patients with advanced liver disease. It should also be noted that we did not evaluate for occult HBV infection, which is defined as HBV DNA in the liver of a patient with negative HBsAg, Occult 
HBV infection may contribute to "cryptogenic" HCC in high prevalence areas such as Taiwan. ${ }^{27}$

In summary, we found that one-third of cryptogenic HCC (most of which is likely related to NAFLD) presented in patients without clinically apparent cirrhosis. Furthermore, these cryptogenic HCC patients were diagnosed at later stages of disease, had larger tumours and had worse overall survival. The epidemiology of non-viral non-alcoholic $\mathrm{HCC}$ is different from that of viral HCC and management guidelines should take this into account as NAFLD becomes an increasingly prevalent risk factor for HCC.

\section{CONFLICT OF INTEREST}

The authors do not have any disclosures to report.

\section{ORCID}

Mindie H. Nguyen (iD http://orcid.org/0000-0002-6275-4989

\section{REFERENCES}

1. Fitzmaurice C, Allen C, Barber RM, et al. Global, regional, and national cancer incidence, mortality, years of life lost, years lived With disability, and disability-adjusted life-years for 32 cancer groups, 1990 to 2015: a systematic analysis for the global burden of disease study. JAMA Oncol. 2016;3:524-548.

2. Chiang C-J, Lo W-C, Yang Y-W, You S-L, Chen C-J, Lai M-S. Incidence and survival of adult cancer patients in Taiwan, 2002-2012. J Formos Med Assoc. 2016;115:1076-1088.

3. Siegel RL, Miller KD, Jemal A. Cancer statistics, 2016. CA Cancer J Clin 2016;66:7-30.

4. Perz JF, Armstrong GL, Farrington LA, Hutin YJF, Bell BP. The contributions of hepatitis $B$ virus and hepatitis $C$ virus infections to cirrhosis and primary liver cancer worldwide. J Hepatol. 2006;45:529-538.

5. Powell EE, Cooksley WG, Hanson R, Searle J, Halliday JW, Powell LW. The natural history of nonalcoholic steatohepatitis: a follow-up study of forty-two patients for up to 21 years. Hepatology. 1990;11:74-80.

6. White DL, Kanwal F, El-Serag HB. Association between nonalcoholic fatty liver disease and risk for hepatocellular cancer, based on systematic review. Clin Gastroenterol Hepatol. 2012;10:1342-1359.

7. Younossi ZM, Stepanova M, Afendy M, et al. Changes in the prevalence of the most common causes of chronic liver diseases in the United States from 1988 to 2008. Clin Gastroenterol Hepatol. 2011;9:524-530; quiz e60.

8. Younossi ZM, Koenig AB, Abdelatif D, Fazel Y, Henry L, Wymer M. Global epidemiology of non-alcoholic fatty liver disease-metaanalytic assessment of prevalence, incidence and outcomes. Hepatology. 2015;64:73-84.

9. Bruix J, Sherman M, American Association for the Study of Liver Diseases. Management of hepatocellular carcinoma: an update. Hepatology. 2011;53:1020-1022.

10. Baffy G, Brunt EM, Caldwell SH. Hepatocellular carcinoma in non-alcoholic fatty liver disease: an emerging menace. J Hepatol. 2012;56:1384-1391.

11. Kawada N, Imanaka K, Kawaguchi T, et al. Hepatocellular carcinoma arising from non-cirrhotic nonalcoholic steatohepatitis. J Gastroenterol. 2009;44:1190-1194.
12. Paradis V, Zalinski S, Chelbi E, et al. Hepatocellular carcinomas in patients with metabolic syndrome often develop without significant liver fibrosis: a pathological analysis. Hepatology. 2009;49:851-859.

13. Tokushige K, Hashimoto E, Horie Y, Taniai M, Higuchi S. Hepatocellular carcinoma in Japanese patients with nonalcoholic fatty liver disease, alcoholic liver disease, and chronic liver disease of unknown etiology: report of the nationwide survey. J Gastroenterol. 2011;46:1230-1237.

14. Mittal S, Sada YH, El-Serag HB, et al. Temporal trends of nonalcoholic fatty liver disease-related hepatocellular carcinoma in the veteran affairs population. Clin Gastroenterol Hepatol. 2015;13:594-601.

15. Piscaglia F, Svegliati-Baroni G, Barchetti A, et al. Clinical patterns of hepatocellular carcinoma in nonalcoholic fatty liver disease: a multicenter prospective study. Hepatology. 2016;63:827-838.

16. Giannini EG, Marabotto E, Savarino V, et al. Hepatocellular carcinoma in patients with cryptogenic cirrhosis. Clin Gastroenterol Hepatol. 2009;7:580-585

17. Margini C, Dufour JF. The story of HCC in NAFLD: from epidemiology, across pathogenesis, to prevention and treatment. Liver Int. 2016;36:317-324.

18. Farrell GC, Larter CZ. Nonalcoholic fatty liver disease: from steatosis to cirrhosis. Hepatology. 2006;43(2 Suppl 1):S99-S112.

19. Bugianesi E, Leone N, Vanni E, et al. Expanding the natural history of nonalcoholic steatohepatitis: from cryptogenic cirrhosis to hepatocellular carcinoma. Gastroenterology. 2002;123:134-140.

20. Ong J, Younossi ZM, Reddy V, et al. Cryptogenic cirrhosis and posttransplantation nonalcoholic fatty liver disease. Liver Transpl. 2001;7:797-801.

21. Simmons O, Fetzer DT, Yokoo T, et al. Predictors of adequate ultrasound quality for hepatocellular carcinoma surveillance in patients with cirrhosis. Aliment Pharmacol Ther. 2017;45:169-177.

22. Kolly P, Dufour J-F. Surveillance for hepatocellular carcinoma in patients with NASH. Diagnostics (Basel). 2016;6: pii: E22.

23. Yasui K, Hashimoto E, Komorizono Y, et al. Characteristics of patients with nonalcoholic steatohepatitis who develop hepatocellular carcinoma. Clin Gastroenterol Hepatol. 2011;9:428-433; quiz e50.

24. Takuma Y, Nouso K. Nonalcoholic steatohepatitis-associated hepatocellular carcinoma: our case series and literature review. World J Gastroenterol. 2010;16:1436-1441.

25. Hsu C-Y, Lee Y-H, Liu P-H, et al. Decrypting cryptogenic hepatocellular carcinoma: clinical manifestations, prognostic factors and longterm survival by propensity score model. PLoS One. 2014;9:e89373.

26. Younossi ZM, Otgonsuren M, Henry L, et al. Association of nonalcoholic fatty liver disease (NAFLD) with hepatocellular carcinoma (HCC) in the United States from 2004 to 2009. Hepatology. 2015;62:1723-1730.

27. Wong DKH, Huang FY, Lai CL, et al. Occult hepatitis B infection and HBV replicative activity in patients with cryptogenic cause of hepatocellular carcinoma. Hepatology. 2011;54:829-836.

\section{SUPPORTING INFORMATION}

Additional Supporting Information may be found online in the supporting information tab for this article.

How to cite this article: Jun TW, Yeh M-L, Yang JD, et al. More advanced disease and worse survival in cryptogenic compared to viral hepatocellular carcinoma. Liver Int. 2018;38:895-902. https://doi.org/10.1111/liv.13613 